\title{
The epidemiology of Neisseria gonorrhoeae isolates in Dakar, Sénégal 1982-1986: antimicrobial resistance, auxotypes and plasmid profiles
}

\author{
I Lind, M Arborio, M W Bentzon, Y Buisson, M Guibourdenche, K Reimann, J-Y Riou
}

\begin{abstract}
A total of 460 Neisseria gonorrhoeae isolates from patients seen at three clinics in Dakar, Sénégal, 1982-1986, have been investigated. In this period a significant change in antimicrobial susceptibility was observed: the percentage of strains susceptible to penicillin (MIC $\leqslant 0.08 \mu \mathrm{g} / \mathrm{ml}$ ) fell from 61 to 18 $(p<0.0001)$ and the percentage of resistant strains (MIC $\geqslant 1.2 \mu \mathrm{g} / \mathrm{ml}$ ) increased from 18 to 46. Among penicillin-resistant strains the proportion of penicillinase-producing strains (PPNG) was fairly constant (range 35-55\%). The determination of susceptibility to antimicrobial agents performed locally allowed detection of approximately all PPNG strains whereas the increase in the occurrence of strains with chromosomally determined resistance was not revealed. The study comprised 70 PPNG strains of which $19 \%(13 / 70)$ carried the $7 \cdot 4 \mathrm{~kb}$ Asian plasmid and $81 \%(57 / 70)$ the $5 \cdot 3 \mathrm{~kb}$ African plasmid. None of these strains possessed the $38 \mathrm{~kb}$ conjugative plasmid, whereas it was found in $4 \cdot 5 \%$ of the 376 non-PPNG strains available for plasmid analysis; $92 \%(410 / 446)$ of all strains had the small $4 \cdot 2 \mathrm{~kb}$ plasmid and $5 \cdot 4 \%(24 / 446)$ did not contain any plasmid. Overall, auxotype zero and proline-requiring strains were predominant, accounting for $53 \%$ $(244 / 460)$ and $28 \%(131 / 460)$, respectively. In general, PPNG strains carrying the $5 \cdot 3 \mathrm{~kb}$ plasmid were auxotype zero $(49 / 57=86 \%)$ and those carrying the $7 \cdot 4 \mathrm{~kb}$ plasmid were prolinerequiring $(9 / 13=69 \%)$.
\end{abstract}

Statens Seruminstitut, Copenhagen, Denmark

I Lind, $M$ W Bentzon, $K$ Reimann

Institut Pasteur, Dakar, Sénégal

M Arborio, Y Buisson

Institut Pasteur, Paris, France

M Guibourdenche, J-Y Riou
Introduction

In many countries the management of gonorrhoea and other sexually transmitted diseases is based on syndrome related treatment algorithms. ${ }^{1}$ An important measure to prevent spread of strains resistant to antibiotics currently in use is a continuous or sentinel surveillance of the in vitro antimicrobial susceptibility of recent clinical isolates of $N$. gonorrhoeae. ${ }^{23}$ The antimicrobial resistance of $N$. gonorrhoeae is rapidly changing, especially in areas where inefficient standard treatment regimens are applied. ${ }^{45}$

The aim of the present study was to describe the evolution in antimicrobial resistance of $N$. gonorrhoeae in Dakar during the period 1982-1986 and to recommend methods suitable for local surveillance of antimicrobial resistance of $N$. gonorrhoeae.

The first part of the study was carried through in 1981-1983, during which period procedures for isolation, identification, transport and storage of $N$. gonorrhoeae was established at the Institut Pasteur in Dakar. ${ }^{6}$ It was in this context that the first penicillinase-producing $N$. gonorrhoeae (PPNG) strain was found in Dakar in $1981 .^{7}$

\section{Materials and methods}

Neisseria gonorrhoeae strains

All gonococcal strains studied were isolated from patients attending three clinics in Dakar, Sénégal, 1982-1986:

Clinic A: The outpatients' clinic at the Institut Pasteur. The majority of patients of both sexes are Africans from lower or middle socio-economic classes.

Clinic B: military personnel, all young European men.

Clinic C: The outpatients" clinic at "Service de Dermatologie de l'Institut d'Hygiene Sociale". Only a few patients attending this clinic were included, except in 1984 when a larger group of female prostitutes were examined.

All isolates were identified, tested for penicillinase ( $\beta$-lactamase) production and, except for 1982 strains, a preliminary antibiogram was determined in Dakar. The isolates were lyophilised and sent to the Institut Pasteur in Paris. 
At the Laboratoire des Neisseria, Institut Pasteur, Paris, the auxotypes were determined according to Catlin ${ }^{8}$ and plasmid analysis was performed on all PPNG strains. The strains were re-lyophilised and approximately 100 strains per year were sent to the Neisseria Department, Statens Seruminstitut, Copenhagen. All strains received at this laboratory were subjected to determination of susceptibility to antimicrobial agents and plasmid analysis (see below).

\section{Antimicrobial susceptibility testing}

Each strain was examined for susceptibility to penicillin, tetracycline and cefotaxime by the agar plate dilution method. ${ }^{9}$ The concentrations of antibiotics using two-fold dilution steps were as follows: sodium penicillin G: $0.01-9.6 \mu \mathrm{g} / \mathrm{ml}$; tetracycline hydrochloride: $0 \cdot 125-32 \mu \mathrm{g} / \mathrm{ml}$; cefotaxime: 0.00025 $1.024 \mu \mathrm{g} / \mathrm{ml}$.

In addition, the susceptibility to penicillin, tetracycline, spectinomycin and thiamphenicol was determined by the agar disc diffusion test using 20 hours pre-diffusion. ${ }^{4}$ The disc content was: penicillin: $2.4 \mu \mathrm{g}$; tetracycline: $10 \mu \mathrm{g}$; spectinomycin: $100 \mu \mathrm{g}$; thiamphenicol: $\quad 30 \mu \mathrm{g}$. The production of $\beta$-lactamase was determined by the chromogenic cephalosporin test. ${ }^{10}$ The WHO $N$. gonorrhoeae reference strains A-D were included on each experimental day (22 experiments).

$N$. gonorrhoeae strains can be divided into three groups according to MIC of penicillin: susceptible: MIC $\leqslant 0.08 \mu \mathrm{g} / \mathrm{ml}$; less susceptible: MIC $0 \cdot 15-0.60 \mu \mathrm{g} / \mathrm{ml} ;$ resistant: $\mathrm{MIC} \geqslant 1 \cdot 2 \mu \mathrm{g} / \mathrm{ml}$. The definition of susceptibility is based on the clinical efficacy of standard treatment regimens. ${ }^{11}$ Ninety five percent of uncomplicated urogenital infections with susceptible strains will be cured by penicillins given in standard dosages, whereas a high cure rate of infections with less susceptible strains can only be achieved by increasing the penicillin dosage. Infections with resistant strains require treatment with alternative drugs.

Table 1 N. gonorrhoeae strains isolated in Dakar 1982-86: prevalence of penicillinase-producing $N$. gonorrhoeae ( $P P N G$ )

\begin{tabular}{llllll}
\hline \multicolumn{5}{c}{ Number of strains isolated } \\
\cline { 2 - 6 } & 1982 & 1983 & 1984 & 1985 & 1986 \\
\hline Clinic A & & & & & \\
$\quad$ Total & 94 & 59 & 61 & 61 & 43 \\
$\quad$ PPNG (\%) & $7(7)$ & $5(8)$ & $8(13)$ & $11(18)$ & $10(23)$ \\
Clinic B & & & & & \\
$\quad$ Total & - & 28 & 25 & 22 & 14 \\
$\quad$ PPNG (\%) & - & $1(4)$ & $5(20)$ & $6(27)$ & $2(14)$ \\
Clinic C & & & & & \\
Total & 4 & 9 & 6 & 34 & - \\
PPNG (\%) & 0 & $3(33)$ & $1(17)$ & $11(32)$ & - \\
Total & 98 & 96 & 92 & 117 & 57 \\
PPNG (\%) & $7(7)$ & $9(9)$ & $14(15)$ & $28(24)$ & $12(21)$ \\
\hline
\end{tabular}

Table 2 Penicillin susceptibility testing of non-penicillinase producing N. gonorrhoeae: Comparison of results obtained by the agar plate dilution method and the agar disc diffusion test (disc content $2 \cdot 4 \mu \mathrm{g}$ pencillin $\mathrm{G}$ )

\begin{tabular}{lcccc}
\hline \multirow{2}{*}{$\begin{array}{l}\text { Zone } \\
\text { diameter } \\
\text { (mm) }\end{array}$} & \multicolumn{4}{l}{ Number of strains at $M I C(\mu \mathrm{g} / \mathrm{ml})$} \\
\cline { 2 - 5 } & $0 \cdot 01-0 \cdot 08$ & $0 \cdot 15-0 \cdot 60$ & $1 \cdot 2-9 \cdot 6$ & Total \\
\hline$\leqslant 35$ & 0 & 4 & 71 & 75 \\
$36-50$ & 12 & 108 & 15 & 135 \\
$\geqslant 51$ & 170 & 9 & 0 & 179 \\
lotal & 182 & 121 & 86 & 389 \\
\hline
\end{tabular}

\section{Plasmid analysis}

The analysis was performed according to the method of Birnboim ${ }^{12}$ in Paris and of Takahashi and Nagano in Copenhagen. ${ }^{13}$

\section{Statistical methods}

Comparisons between frequencies are carried out using Fisher's exact test. Product-moment correlation coefficients (denoted $r$ ) are used to characterise the interdependence between MICs for different antibiotics and between MICs and zone diameters.

\section{Results}

\section{Study population}

The 460 gonococcal strains received in Copenhagen originated from 318 patients seen at clinic $A$ ( 249 men and 69 women), 89 patients seen at clinic $B$ (all men) and 53 patients seen at clinic C (19 men in 1982-84 and 34 women in 1985).

\section{Prevalence of penicillinase-producing $N$. gonorrhoeae (PPNG).}

As shown in table 1 , a steady increase of PPNG infections was registered at clinic $\mathrm{A}$, namely from $7 \%$ in 1982 to $23 \%$ in 1986 . This change is statistically significant $(p=0 \cdot 01)$. The changes seen at the other two clinics probably reflect the same evolution, but the figures are too small to be subjected to a detailed analysis. Overall 70 or $15 \%$ of the gonococcal strains recovered were PPNG.

\section{Susceptibility to penicillin}

A comparison of the results obtained for 69 PPNG and 389 non-PPNG strains by the agar dilution method (MIC in $\mu \mathrm{g} / \mathrm{ml}$ ) and the agar disc diffusion test (zone diameters in $\mathrm{mm}$ ) was carried out (table 2). All PPNG strains showed maximum MICs of penicillin (not shown) and the inhibition zone for 67 out of 69 strains was zero whereas two strains showed a small zone $(<15 \mathrm{~mm})$ in the experiments recorded. For 389 non-PPNG strains a strong correlation between MIC and zone diameter was found $(r=$ $-0.93)$ and $22 \%(86 / 389)$ had high level chromo- 
somally mediated resistance to penicillin (MIC $\geqslant 1.2 \mu \mathrm{g} / \mathrm{ml}$ ); of these CMRNG (chromosomal mediated resistant $N$. gonorrhoeae) strains 11 had MICs $\geqslant 4.8 \mu \mathrm{g} / \mathrm{ml}$ and exhibited no zone of inhibition by the agar disc diffusion test.

The three levels of susceptibility to penicillin defined by means of MICs (see Materials and methods) can also be determined by the agar disc diffusion test. As shown in Table 2, 93\% (170/182) of penicillin-susceptible strains had an inhibition zone diameter $\geqslant 51 \mathrm{~mm}, 89 \%(108 / 121)$ of less susceptible strains a zone diameter between 36 and $50 \mathrm{~mm}$, and $83 \%(71 / 86)$ of resistant, non-PPNG strains a zone diameter $\leqslant 35 \mathrm{~mm}$. None out of the 182 penicillin-susceptible strains showed zone diameters $\leqslant 35 \mathrm{~mm}$ and none out of the 86 penicillin-resistant strains showed zone diameters $\geqslant 51 \mathrm{~mm}$. If these findings are used as a basis for definition of zone diameters corresponding to the three different levels of susceptibility, the classifications by the two methods disagree for 40 strains or about $10 \%$, the strains being placed in groups adjacent to the correct one.

\section{Susceptibility to tetracycline}

A comparison of results obtained by the agar plate dilution method and the agar disc diffusion test using a disc containing $10 \mu \mathrm{g}$ tetracycline hydrochloride was also performed. There was a strong correlation between the results obtained by the two methods $(\mathrm{r}=-0.87) ; 32 \%(149 / 458)$ were susceptible, $58 \%$ $(265 / 458)$ were less susceptible (MIC $=1 \cdot 0-4 \cdot 0$ $\mu \mathrm{g} / \mathrm{ml})$ and $10 \%(44 / 458)$ were resistant $(\mathrm{MIC} \geqslant 8.0 \mu \mathrm{g} / \mathrm{ml}$ ) to tetracycline. For non-PPNG strains there was a strong correlation between MICs of penicillin and of tetracycline $(r=0 \cdot 80)$. Susceptible (zone diameter $\geqslant 41 \mathrm{~mm}$ ) and resistant (zone diameter $\leqslant 30 \mathrm{~mm}$ ) strains were clearly recognised by means of the agar disc diffusion test. If these findings are used as a basis for definition of three levels of susceptibility to tetracycline by means of the agar disc diffusion test, it was found that 83 out of $\mathbf{4 5 8}$ strains or $18 \%$ showed a one-level discrepancy when a comparison with the corresponding MIC levels was made.

\section{Susceptibility to cefotaxime}

There was a strong correlation between MICs of penicillin and cefotaxime of non-PPNG strains $(r=0.94)$. The MIC of cefoxatime was within the range $0.005-0.512 \mu \mathrm{g} / \mathrm{ml}$; out of the 86 CMRNG strains $32(37 \%)$ showed a MIC of cefotaxime of $0.064 \mu \mathrm{g} / \mathrm{ml}$ and $24(28 \%)$ showed a MIC of cefotaxime of $0.128 \mu \mathrm{g} / \mathrm{ml}$ or greater. The corresponding numbers (percentages) of the 70 PPNG strains were $4(6 \%)$ and $3(4 \%)$, respectively.
Table 3 Penicillin susceptibility of non-penicillinase producing N. gonorrhoeae strains isolated in Dakar 1982-86

\begin{tabular}{lllll}
\hline & & \multicolumn{4}{c}{ Percentage of strains at MIC of penicillin $(\mu \mathrm{g} / \mathrm{ml})$} \\
\cline { 3 - 5 } Year & $N$ & $0.01-0.08$ & $0 \cdot 15-0.60$ & $1 \cdot 2-9 \cdot 6$ \\
\hline 1982 & 91 & 66 & 22 & 12 \\
1983 & 87 & 57 & 23 & 20 \\
1984 & 78 & 38 & 36 & 26 \\
1985 & 89 & 37 & 36 & 27 \\
1986 & 45 & 22 & 47 & 31 \\
\hline
\end{tabular}

\section{Susceptibility to spectinomycin}

All strains were susceptible to spectinomycin; $95 \%$ (437/460) showed a zone of inhibition within the limits 26-35 mm corresponding to MICs at $\leqslant 16 \mu \mathrm{g} / \mathrm{ml}$ (unpublished data). There was no difference between PPNG and non-PPNG strains, including CMRNG strains.

\section{Susceptiblity to thiamphenicol}

In agreement with previous findings a positive correlation between susceptibility to thiamphenicol and to penicillin could be demonstrated in nonPPGN strains (data not shown). For thiamphenicol a strong correlation between MICs and zone diameter has been demonstrated previously (Spearman Rank correlation coefficient, -0.77$){ }^{4}$

Changes in antimicrobial susceptibility of $N$. gonorrhoeae strains isolated in Dakar 1982-1986

Table 3 shows the percentage of non-PPNG strains at each level of MIC of penicillin during the period 1982-1986. The percentage of penicillin-susceptible strains fell from $66 \%$ in 1982 to $22 \%$ in 1986 $(\mathrm{p}<0.0001)$ and during the same period the percentage of resistant strains increased from $12 \%$ to $31 \%$. The figure illustrates changes in the prevalences of PPNG and CMRNG strains and of strains less susceptible and fully susceptible to penicillin. The increase in the prevalence of penicillin-resistant strains reflects a spread of PPNG as well as of CMRNG strains.

Comparison of results of antimicrobial susceptibility testing obtained in Dakar and in Copenhagen

Out of 272 strains that had been examined both in Dakar and Copenhagen, 72 had been recorded as penicillin-resistant in Dakar. Of these $\mathbf{7 2}$ strains $\mathbf{5 0}$ were designated PPNG; in Copenhagen 54 out of the 272 strains were found to be PPNG, that is, the recovery rate in Dakar was $93 \%$ (50/54). Out of the 272 strains 63 were CMRNG. In Dakar 22 were recorded as penicillin-resistant non-PPNG strains; only 11 of these 22 strains were actually CMRNG, which means that $83 \%(52 / 63)$ of CMRNG strains remained unrecognised. 


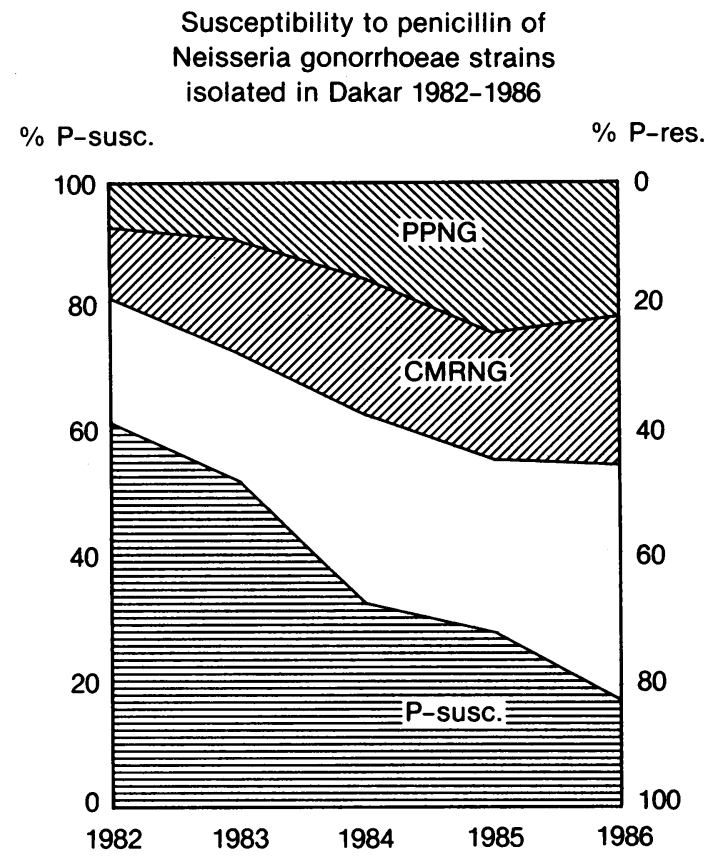

Figure Observed changes in the percentages of PPNG, $C M R N G$, and of gonococcal strains fully susceptible or less susceptible (blank area) to penicillin isolated in Dakar during the period 1982-1986.

Correlation between auxotype and susceptibility to penicillin

The proline-requiring and the proline plus argininerequiring non-PPNG strains showed the same distribution according to susceptibility to penicillin, $48 \%$ and $54 \%$ of the strains being penicillinresistant, respectively (table 4). These two groups were significantly more resistant than auxotype zero gonococci $(p<0.0001)$ and than arginine-requiring strains $(p=0.007)$; arginine-requiring strains and the mixed group of strains showed a distribution similar to that of auxotype zero strains. In general, auxotype zero PPNG strains carried the $5.3 \mathrm{~kb}$ plasmid $(49 / 52=94 \%)$ and proline-requiring PPNG carried the $7 \cdot 4 \mathrm{~kb}$ plasmid $(9 / 11=82 \%)$.

Correlation between plasmid content and susceptibility to penicillin

The distribution according to susceptibility to penicillin for non-PPNG strains containing none $(\mathrm{N}=24)$, the $4.2 \mathrm{~kb}(\mathrm{~N}=326)$ or the $11.8 \mathrm{~kb}$ $(\mathrm{N}=3)$ cryptic plasmids $^{14}$ or the 4.2 plus the $11.8 \mathrm{~kb}$ plasmids $(\mathrm{N}=6$ ) were similar (table 5 ). Strains containing the $38 \mathrm{~kb}$ conjugative plasmid alone $(\mathrm{N}=9)$ were more susceptible to penicillin than those carrying the small cryptic plasmid $(4 \cdot 2 \mathrm{~kb})$ alone $(p=0.002)$ whereas those strains carrying both plasmids $(\mathbf{N}=8)$ are more often CMRNG $(p=0.028)$.
Table 4 N. gonorrhoeae strains isolated in Dakar 1982-86: Correlation between auxotype and susceptibility to penicillin

\begin{tabular}{lcccccc}
\hline \multicolumn{7}{c}{$\begin{array}{l}\text { Number of non-PPNG strains at MIC } \\
(\mu \mathrm{g} / \mathrm{ml})\end{array}$} \\
\cline { 2 - 6 } Auxotype & $0 \cdot 01-0.08$ & $0 \cdot 15-0 \cdot 60$ & $1 \cdot 2-9 \cdot 6$ & Total & PPNG & Total \\
\hline Zcro & 116 & 67 & 9 & 192 & 52 & 244 \\
Pro & 25 & 38 & 57 & 120 & 11 & 131 \\
Arg & 16 & 1 & 3 & 20 & 0 & 20 \\
Pro Arg & 6 & 7 & 15 & 28 & 1 & 29 \\
Others & 20 & 8 & 2 & 30 & 6 & 36 \\
Total & 183 & 121 & 86 & 390 & 70 & 460 \\
\hline
\end{tabular}

Prevalence of gonococcal strains containing the $5.3 \mathrm{~kb}$, the $7.4 \mathrm{~kb}$ or the $38 \mathrm{~kb}$ conjugative plasmid

The study comprised 70 PPNG strains of which $19 \%$ $(13 / 70)$ carried the $7.4 \mathrm{~kb}$ plasmid and $81 \%(57 / 70)$ the $5.3 \mathrm{~kb}$ plasmid. None of these strains possessed the $38 \mathrm{~kb}$ plasmid, whereas it was found in $4.5 \%$ (17/376) of non-PPNG strains; $92 \%(410 / 446)$ of all strains, including all PPNG strains, had the small $4.2 \mathrm{~kb}$ plasmid and $5.4 \%(24 / 446)$ did not contain any plasmid.

Correlation between auxotype and plasmid content Results of auxotyping and plasmid analysis were available for $\mathbf{4 4 6}$ strains. Auxotype zero and prolinerequiring strains were predominant (table 4 ); $86 \%$ $(49 / 57)$ of PPNG strains carrying the $5.3 \mathrm{~kb}$ plasmid were auxotype zero and $69 \%$ (9/13) of PPNG strains carrying the $7.4 \mathrm{~kb}$ plasmid were proline-requiring. Arginine-requiring and arginine- plus prolinerequiring strains were uncommon, and only one of these strains contained the $5.3 \mathrm{~kb}$ plasmid.

\section{Discussion}

The first isolate of penicillinase-producing N. gonorrhoeae (PPNG) in Africa was reported in 1979, and two years later, by 1981, reports from 23 African countries documented the presence of PPNG infections. ${ }^{15}$ There is indirect evidence that in certain areas the PPNG strains had emerged earlier, prob-

Table 5 Non-penicillinase producing $N$. gonorrhoeae strains isolated in Dakar 1982-86: Correlation between plasmid content and susceptibility to penicillin

\begin{tabular}{lcccr}
\hline & \multicolumn{4}{c}{ Number of strains at MIC $(\mu \mathrm{g} / \mathrm{ml})$} \\
\cline { 2 - 5 } Plasmid content & $0.01-0.08$ & $0.15-0.60$ & $1 \cdot 2-9.6$ & Total \\
\hline None & 13 & 3 & 8 & 24 \\
$38 \mathrm{~kb}$ & 9 & 0 & 0 & 9 \\
$11.8 \mathrm{~kb}$ & 2 & 0 & 1 & 3 \\
$4 \cdot 2 \mathrm{~kb}$ & 149 & 109 & 68 & 326 \\
$4.2 \mathrm{~kb}+38 \mathrm{~kb}$ & 1 & 2 & 5 & 8 \\
$4 \cdot 2 \mathrm{~kb}+11.8 \mathrm{~kb}$ & 4 & 1 & 1 & 6 \\
Total & 178 & 115 & 83 & 376 \\
\hline
\end{tabular}


ably in $1976 .{ }^{16}$ During the following years, PPNG strains spread all over Africa, but with different trends; in Ibadan, Nigeria, for example, the prevalence of PPNG infection had reached $81 \%$ in $1984,{ }^{15}$ whereas in the present study from Dakar, Sénégal, the rate reached only $23 \%$ up to the year 1986. In a semirural community in Gabon, PPNG infections accounted for $7 \%$ of cases of gonorrhoea in $1981,48 \%$ in 1984 and then only $23 \%$ in $1985 .{ }^{17}$

At the emergence of PPNG strains, two distinct types of strains were recognised: one type contained a $5.3 \mathrm{~kb} \beta$-lactamase-coding plasmid, did not contain the $38 \mathrm{~kb}$ transfer plasmid, required ärginine $\left(\mathrm{Arg}^{-}\right)$ for growth, was susceptible to tetracycline and epidemiologically linked to West Africa; another type contained a $7.4 \mathrm{~kb} \beta$-lactamase-coding plasmid, and, in addition, around $40 \%$ contained the $38 \mathrm{~kb}$ transfer plasmid; these strains were auxotype zero (wild type) or proline-requiring, tetracyclineresistant, and epidemologically linked to the Far East. Since then, the $\beta$-lactamase-coding plasmids have spread among different gonococcal auxotypes and among strains with different antimicrobial susceptibility patterns; $;^{18-20}$ the $38 \mathrm{~kb}$ transfer plasmid has been found also in strains carrying the $5.3 \mathrm{~kb}$ plasmid and in varying proportions of non-PPNG strains. The spread of $\beta$-lactamase-coding plasmids seems to be independent of the presence of the $38 \mathrm{~kb}$ plasmid, and this latter plasmid has spread rapidly in certain gonococcal populations. ${ }^{21}$

In Dakar the predominant type of PPNG during the period 1982-1986 was auxotype zero carrying the $5.3 \mathrm{~kb}$ plasmid, of which one carried the $38 \mathrm{~kb}$ plasmid. Overall, auxotype zero $(53 \%)$ and prolinerequiring $(28 \%)$ strains were predominant, and strains that required arginine or arginine as well as proline were uncommon (4\% and $6 \%$, respectively). The $38 \mathrm{~kb}$ plasmid was detected in a few strains every year and showed no tendency to spread; in total, this plasmid was only found in $4.5 \%$ of the non-PPNG strains studied. This finding is in contrast to the results of a contemporary study, in which the prevalence of the $38 \mathrm{~kb}$ plasmid in isolates from Greenland was found to increase from $0 \%$ to $70 \%$ (Reimann K and Lind I: unpublished data), Knapp has reported on a similar rapid spread in some areas of the U.S.A. ${ }^{21}$

The spread of penicillin-resistant non-PPNG (CMRNG) strains in Africa has not been studied as closely as the spread of PPNG. In the report from Gabon, ${ }^{17}$ the percentage of isolates for which the MIC of tetracycline was greater than $1 \mu \mathrm{g} / \mathrm{ml}$ increased from $5 \%$ in 1981 or 1984 to $28 \%$ in 1985 ; this finding may reflect an increased importation of CMRNG strains, for example from the Far East, although the MIC of penicillin for $90 \%$ of isolates passed from $2 \mu \mathrm{g} / \mathrm{ml}$ in 1981 to $0.5 \mu \mathrm{g} / \mathrm{ml}$ in 1985 . In our study, a significant change in antimicrobial sus- ceptibility of non-PPNG-strains was observed: the percentage of CMRNG strains increased from 12 in 1982 to 31 in 1986, and the percentage of susceptible strains fell from 66 to 22 ( $p<0.0001)$. The possibility of detecting these changes by antimicrobial susceptibility testing performed locally was assessed. The local performance allowed detection of approximately all PPNG strains, whereas the increase in the occurrence of strains with chromosomally determined resistance was not revealed. In retrospect, details of the agar disc diffusion test used, conditions for storage of discs, definitions used for the designations $\mathbf{r}$ (resistance) versus $\mathbf{s}$ (susceptible), etc. could not be cleared up adequately. It is considered most likely that inappropriate storage conditions of the penicillincontaining discs were the reason why a high proportion of CMRNG strains remained unrecognised.

In the reference laboratory in Copenhagen, the results obtained by the agar plate dilution method and by agar disc diffusion tests were in agreement, and both methods allowed a current assessment of changes in antimicrobial pattern. Each of the 22 experiments included the WHO $N$. gonorrhoeae reference strains A-E. Strain E is a PPNG. There was a strong correlation between MIC and zone diameter, and the four different levels of penicillin susceptibility defined by the reference strains $A-D$ could be identified by both methods without any overlapping. The study from Gabon included the WHO reference strains A-E in each series of MIC determinations (14 experiments). The different levels of susceptibility to penicillin (and other antibiotics) were correctly identified, and only $4 \cdot 2 \%$ (14 of 336 ) of the determinations differed by more than one dilution from the median. Therefore, the above-mentioned results obtained in two studies of African strains are directly comparable and provide an example of the benefit of using reference strains.

In general, disc diffusion tests are performed without pre-diffusion, that is, without establishing a concentration gradient of the antibiotic into the medium before inoculation; regarding $\beta$-lactam antibiotics and tetracycline, discs with higher contents than those employed in the present study are commercially available and therefore most often used. Under these conditions, the differences between diameters of inhibition zones for susceptible, less susceptible and resistant strains decrease, especially if the medium is unfavourable to growth of gonococci (Lind I and Bentzon M W: unpublished data). If the observed differences for the reference strains are less than the expected day-to-day variation, the disc diffusion test is of no use. When classification into three groups is made on the basis of results obtained by two different methods, complete agreement cannot be expected in spite of the strong correlation found between the two sets of results. The findings of $90 \%$ 
(penicillin) and $82 \%$ (tetracycline) agreement between MICs and zone diameter, and the fact that none of the susceptible strains were categorised as resistant and vice versa, indicate that the agar disc diffusion method is useful.

Snell and Brown ${ }^{22}$ organised an external quality assessment of antimicrobial susceptibility testing of $N$. gonorrhoeae comprising 411 United Kingdom laboratories. Detection of PPNG was achieved by almost $100 \%$ of the laboratories, whereas the detection of CMRNG proved more difficult. They demonstrated that greater error rates were associated with the use of high content discs for testing tetracycline $(25,30$ or $50 \mu \mathrm{g}$ versus 2,5 or $10 \mu \mathrm{g})$ and with the use of low content discs for testing spectinomycin (25 or $30 \mu \mathrm{g}$ versus $100 \mu \mathrm{g}$ ). Unfortunately, an insufficient number of laboratories used cefuroxime discs other than $30 \mu \mathrm{g}$ to allow comparison to be made. There was no difference in error rates using the following low contents of penicillin: $1,1.5$ and 2 units $(0.6,0.9$ and $1.2 \mu \mathrm{g})$ per disc. These results are in complete agreement with those obtained in our laboratories. The recent comprehensive study from the USA ${ }^{23}$ on the standardisation of antimicrobial susceptibility testing of $N$. gonorrhoeae focuses on lot to lot variations for commercially available discs (one high content disc per antibiotic) and for components of the media, and on the stability of antibiotics in agar plates. The interpretive criteria recommended for the agar disc diffusion test were derived from results using high content discs and are thus not comparable with those obtained in the present study. The importance of the choice of medium for the antimicrobial susceptibility testing of $N$. gonorrhoeae has also been emphasised by Gill and Ison, ${ }^{24}$ who included the WHO as well as other reference strains in their study.

As discussed by Judson, ${ }^{25}$ the guidelines "Antibiotic-Resistant Strains of Neisseria gonorrhoeae, Policy Guidelines for Detection, Management, and Control" published by Centers for Disease Control in the USA ${ }^{26}$ present extremely complex and detailed recommendations which in certain cases lack supporting data. This is also true for the section on determination of antimicrobial susceptibility. Reservations on recommendations based on limited data are clearly stated in the preamble of the recommendations. For that reason, the obvious discrepancies between our conclusions and those from the CDC will not be discussed in detail.

Our study has confirmed that the use of welldefined reference strains is a valuable tool in the standardisation of antimicrobial susceptibility testing of gonococci. It has also shown that it is possible to identify accurately the various levels of antibiotic susceptibility of $N$. gonorrhoeae by means of the agar disc diffusion test. In other words, the agar disc diffusion test can be standardised and used as an equivalent to the agar plate dilution test in the surveillance of the antimicrobial susceptibility of $N$. gonorrhoeae.

This work was supported by EEC contracts No. TSD-M-109-DK and No. TSD-M-012-F.

Address for correspondence: Inga Lind, MD, Dr sci, Neisseria Department, Statens Seruminstitut Artillerivej 5, DK-2300 Copenhagen S, Denmark.

1 Meheus A. Practical approaches in developing countries. In: Holmes KK, Mårdh PA, Sparling PF, Wiesner PJ, eds. Sexually transmitted diseases. New York: McGraw-Hill, 1984:998-1008.

2 WHO Scientific Group. Neisseria gonorrhoeae and gonococcal infection. WHO Tech Rep Ser 1978; No. 616.

3 Centers for Disease Control. Sentinel surveillance system for antimicrobial resistance in clinical isolates of Neisseria gonorrhoeae. MMWR 1987;36:585-93.

4 Lind I. In vitro susceptibility of Neisseria gonorrhoeae to thiamphenicol: Results for selected groups of strains from different geographic areas and from different times. Sex Transm Dis 1984;Suppl 11:360-3.

5 Lind I. Review. Present status of antimicrobial resistance of Neisseria gonorrhoeae. Eur J Sex Transm Dis 1986;3:185-9.

6 Buisson Y, Guibourdenche M, Renault-Steens C, Riou JY. Approche épidémiologique des gonococcies au Sénégal par l'étude des auxotypes. Bull Soc Pathol Exot Filiales 1984;77:617-27.

7 Buisson Y, Riou JY, Labidi A, Arborio M, Guibourdenche M. A propos des gonocoques producteurs de beta-lactamase isolés à Dakar. Sensibilité à divers antibiotiques, auxotype et contenu plasmidique. Path Biolol (Paris) 1985;33:550-3.

8 Catlin BW. Nutritional profiles of Neisseria gonorrhoeae, Neisseria meningitidis and Neisseria lactamica in chemically defined media and the use of growth requirements for gonococcal typing. J Infect Dis 1973;128:178-94.

9 Reyn A, Bentzon MW, Ericsson H. Comparative investigations of the sensitivity of $N$. gonorrhoeae to penicillin. Acta Path Microbiol Scand 1963;57:235-55.

10 O'Callaghan CH, Morris A, Kirby SM, Shingler AH. Novel method for detection of $\beta$-lactamases by using a chromogenic cephalosporin substrate. Antimicrob Agents Chemother 1972; 1:283-8.

11 WHO Consultative Group. Current treatments in the control of sexually transmitted diseases. Working document. WHO/ VDT 1983;83:433.

12 Birnboim HC, Doly J. A rapid alkaline extraction procedure for screening recombinant plasmid DNA. Nucl Acid Res 1979; 7:1513-23.

13 Takahashi $S$, Nagano Y. Rapid procedure for isolation of plasmid DNA and application to epidemiological analysis. $J$ Clin Microbiol 1984;20:608-13.

14 Johnson SR, Anderson BE, Biddle JW, Perkins GH, DeWitt WE. Characterization of concatemeric plasmids of Neisseria gonorrhoeae. Infect Immun 1983;40:843-6.

15 Osoba AO. Overview of penicillinase producing Neisseria gonorrhoeae in Africa. Afr J STD; Oct 1986:51-64.

16 Perine PL, Thornsberry C, Schalla W, et al. Evidence for two distinct types of penicillinase-producing Neisseria gonorrhoeae. Lancet 1977;ii: 993-5.

17 Peeters M, Frost EH, Collet M, Ossari S, Yvert F, Ivanoff B. Changing antibiotic susceptibility of Neisseria gonorrhoeae in Franceville, Gabon. Antimicrob Agents Chemother 1987; 31:1288-90.

18 Perine PL, Totten PA, Knapp JS, Holmes KK, Bentsi C, Klufio CA. Diversity of gonococcal plasmids, auxotypes, and serogroups in Ghana. Lancet 1983;i:1051-2.

19 Odugbemi TO, Brown ST, Biddle J, et al. Plasmid profile, serogrouping, and auxotyping of Neisseria gonorrhoeae isolates from Africa. Br J Venereal Dis 1983;59:41-3.

20 Ansink-Schipper MC, van Klingeren B, Huikeshoven $M H$, Woudstra RK, Dessens-Kroon M, van Wijngaarden LJ. Epidemiology of PPNG infections in the Netherlands: 
Analysis by auxanographic typing and plasmid identification. Br J Venereal Dis 1984;60:141-6.

21 Knapp JS, Zenilman JM, Rice RJ, Roberts MC, McIntire SA, Morse SA. Frequency in the United States of non- $\beta$ lactamase-producing strains of Neisseria gonorrhoeae possessing the 24.5-Mdal conjugative plasmid. Sex Transm Dis 1989;16:79-83.

22 Snell JJS, Brown DFJ. Antimicrobial susceptibility testing of Neisseria gonorrhoeae: a trial organised as part of the United Kingdom national external quality assessment scheme for microbiology. J Clin Microbiol 1988;41:97-102.

23 Jones RN, Gavan TL, Thornsberry C, et al. Standardization of disk diffusion and agar dilution susceptibility tests for Neisseria gonorrhoeae: Interpretive criteria and quality control guidelines for ceftriaxone, penicillin, spectinomycin and tetracycline. J Clin Microbiol 1989;27:2758-66.

24 Gill MJ, Ison CA. Susceptibility testing of Neisseria gonorrhoeae to penicillin and spectinomycin in a diagnostic laboratory. $J$ Clin Pathol 1988;41:978-82.

25 Judson FN. Management of antibiotic-resistant Neisseria gonorrhoeae. Ann Intern Med 1989;110:5-7.

26 Centers for Disease Control. Antibiotic-resistant strains of Neisseria gonorrhoeae. Policy guidelines for detection, management, and control. MMWR Suppl 1987;36/5S:1-18.

Accepted for publication 17 December 1990 